\title{
Temporal changes of quantitative CT findings from 102 patients with COVID-19 in Wuhan, China: A longitudinal study
}

\author{
Xiaohui Chen ${ }^{\mathrm{a}, 1}$, Wenbo Sun ${ }^{\mathrm{a}, 1}$, Dan Xu ${ }^{\mathrm{b}}$, Jiaojiao $\mathrm{Ma}^{\mathrm{a}}$, Feng Xiao ${ }^{\mathrm{a}}$ and Haibo $\mathrm{Xu}^{\mathrm{a}, *}$ \\ ${ }^{a}$ Department of Radiology, Zhongnan Hospital of Wuhan University, Wuhan, Hubei 430071, China \\ ${ }^{\mathrm{b}}$ Medical Imaging Laboratory, Wuhan University, Wuhan, Hubei 430071, China
}

\begin{abstract}
.
BACKGROUND: Computed tomography (CT) imaging combined with artificial intelligence is important in the diagnosis and prognosis of lung diseases.

OBJECTIVE: This study aimed to investigate temporal changes of quantitative CT findings in patients with COVID-19 in three clinic types, including moderate, severe, and non-survivors, and to predict severe cases in the early stage from the results. METHODS: One hundred and two patients with confirmed COVID-19 were included in this study. Based on the time interval between onset of symptoms and the CT scan, four stages were defined in this study: Stage-1 ( $0 \sim 7$ days); Stage-2 ( $8 \sim 14$ days); Stage-3 (15 21days); Stage-4 (> 21 days). Eight parameters, the infection volume and percentage of the whole lung in four different Hounsfield (HU) ranges, $((-,-750),[-750,-300),[-300,50)$ and $[50,+))$, were calculated and compared between different groups.

RESULTS: The infection volume and percentage of four HU ranges peaked in Stage-2. The highest proportion of HU [ -750 , 50) was found in the infected regions in non-survivors among three groups.

CONCLUSIONS: The findings indicate rapid deterioration in the first week since the onset of symptoms in non-survivors. Higher proportion of $\mathrm{HU}[-750,50)$ in the lesion area might be a potential bio-marker for poor prognosis in patients with COVID-19.
\end{abstract}

Keywords: COVID-19, quantitative CT parameters, temporal changes, early detection

\section{Introduction}

Since December 2019, a novel coronavirus, severe acute respiratory syndrome coronavirus 2 (COVID19), was reported [1,2] and has rapidly spread to most countries in the world. By July 242020 , the number of confirmed COVID-19 cases worldwide had risen above 15 million, and the death toll from COVID-19 passed 619,150 worldwide, equivalent to a mortality rate of around $4.13 \%$ [3].

COVID-19 has a high mortality rate, and it progresses rapidly without obvious signs. In previous studies, the times between onset of symptoms and the emergence of ARDS could be as short as 9 days [4]. Despite real-time reverse transcription polymerase chain reaction (RT-PCR) is used as one of the main tool for diagnosing COVID-19 [5], chest computed tomography (CT) imaging, as an auxiliary mean

\footnotetext{
${ }^{1}$ These two authors contributed equally to the article.

${ }^{*}$ Corresponding author: Haibo Xu, Department of Radiology, Zhongnan Hospital of Wuhan University, Wuhan, Hubei 430071, China. Tel.: +86 2767812533; Fax: +86 2767812533; E-mail: xuhaibo1120@ hotmail.com.
} 
of examination, is fast and can produce fast diagnosis for suspected patients with COVID-19. Because of its high efficiency and sensitivity, CT imaging diagnosis report was once as one of the COVID19 clinical diagnostic criterion in the Hubei Province by the Chinese Center for Disease Control and Prevention [6]. However, there are individual differences in observing the infection regions among radiologists. Therefore, a combination of artificial intelligence (AI) and CT imaging could be helpful in the diagnosis and prognosis of COVID-19 [7,8].

However, at present, there is no research on how to evaluate the severity from temporal changes of the quantitative CT findings in detail at the early stage $[9,10]$. In this study, we extracted the quantitative parameters associated with lesions from CT images and investigated temporal changes of 8 quantitative parameters in patients with COVID-19, and compared the quantitative parameters across the disease course between three clinic types patients (moderate $\backslash$ severe $\backslash$ non-survivors), aimed to screen severe cases and predict the outcome in the early stage.

\section{Materials and methods}

\subsection{Patients}

This retrospective study included 102 adult inpatients at Zhongnan Hospital of Wuhan University (Wuhan, China), who died or were discharged between Jan 1, 2020 and Mar 1, 2020. The requirement for informed consent was waived by the ethics commission for this retrospective study. The median age of the 102 patients was 56.0 years (IQR 41.0-68.0), ranging from 25 years to 96 years.

\subsection{Inclusion and exclusion criterions}

All patients diagnosed with COVID-19, confirmed by real-time RT-PCR analysis of throat swab specimens, with obvious lesions in their CT images were included. The patients whose RT-PCR results were positive but CT results normal were exclude.

\subsection{Typing}

Following the guideline of COVID-2019 (trial version 7) issued by the China National Health Commission, the severity includes four types: mild, common, severe, and critical [11]. In order to distinguish patients discharged or not, we categorized patients to three groups based on the severity: moderate (patients with mild symptoms, including mild and common), severe (survivors with severe symptoms, including severe and critical) and non-survivors. Mild symptoms were defined as not meeting the following three criteria and severe symptoms were defined as meeting one of the following criteria during the disease course: 1) severe respiratory distress (respiratory rate $>30$ breaths/min); 2) Requirement for oxygen treatment support; 3) SpO2 $<93 \%$ in the resting state. Survivors with severe symptoms were discharged of severe symptoms and non-survivors were people who were died of severe symptoms. Patients were assigned to three groups: 43 (42\%) patients had mild symptoms (the moderate group), 39 (38\%) patients were discharged of severe symptoms (the severe group) and the remaining 20 (20\%) died (the non-survivors group).

\subsection{Stages}

Based on the time interval between onset of symptoms and the CT scan, we designated four stages in 
our study: Stage-1 (CT scans done $\leqslant 7$ days after symptoms onset); Stage- 2 ( 8 days $\leqslant$ CT scans done $\leqslant$ 14 days after symptoms onset); Stage- 3 ( 15 days $\leqslant$ CT scans done $\leqslant 21$ days after symptoms onset); Stage-4 (CT scans done $>21$ days after symptoms onset). Quality control of CT images was checked by two radiologists with several years of experience.

\section{CT image analyses}

\subsection{Image acquisition}

All CT scans were obtained with patients in the supine position, using GE discover CT750 (GE Healthcare, USA). Patients were scanned from the lever of the upper thoracic inlet to the inferior lever of the costophrenic angle. For CT acquisition, the tube voltage was $120 \mathrm{kVp}$ and the tube current was modulated by automatic exposure control system. Images were reconstructed with a slice thickness of $1 \mathrm{~mm}$ from the raw data, respectively. The reconstructed images were transmitted to the workstation and picture archiving and communication systems (PACS) for post-processing.

\subsection{Lesion segmentation}

An artificial intelligence (AI) software, uAI-Discover-PNA (pneumonia) [12], developed by United Imaging Intelligence Co., Ltd, was used in the image analysis. The segmentation algorithm in the software is based on a modified 3-D convolutional neural network called VB-net [12]. The VB-net adopted the bottle-neck blocks during the down-sampling and up-sampling operations inside the V-shaped network (V-net) [12]. The bottle-neck blocks use a stacked 3-layer structure $(1 \times 1 \times 1,3 \times 3 \times 3$ and $1 \times 1 \times 1$ convolution kernels), which could help reduce and combine feature map channels, and makes the VB-net faster than the V-net in dealing with large 3D volumetric data [12]. Besides, a human-in-the-loop strategy was applied to iteratively update the VB-net segmentation network via a human-machine interaction manner [12]. The segmentation results produced by the VB-net would be corrected by the radiologist, and then the corrected segmentation results were fed into the model as new training data, which could improve the performance of the deep learning model after 3 iterations [12]. The VB-net could simultaneously segment five lung lobes, 18 lung segments and lung lesions, and yields a Dices index of 0.916 [13] between automatic and manual segmentations when validated on 300 COVID-19 subjects [12].

\subsection{Image parameters extraction}

According to the segmentation results, 8 parameters, which were potentially related to COVID-19, the infection volume and percentage of $\mathrm{HU}(-,-750), \mathrm{HU}[-750,-300), \mathrm{HU}[-300,50), \mathrm{HU}[50,+)$ in all lung were calculated. The infection volume and percentage of the whole lung, each lung lobe and segments were calculated in four HU ranges as following:

$$
\begin{aligned}
& \text { Infection volume }(\mathrm{x})=\mathrm{V}\left(\mathrm{x} \_ \text {infection }\right) \\
& \text { Infection percentage }=\frac{\mathrm{V}\left(\mathrm{x} \_ \text {infection }\right)}{\mathrm{V}(\mathrm{x})}
\end{aligned}
$$

Where $\mathrm{V}($.) represents the volume of the region of interest, $\mathrm{x}$ represents different lung lobes or segments, and $\mathrm{x}$ _infection represents the infection region in $\mathrm{x}$ [13]. 
S300 X. Chen et al. / Temporal changes of quantitative CT findings from 102 patients with COVID-19 in Wuhan, China

Table 1

Baseline characteristics

\begin{tabular}{|c|c|c|c|c|c|}
\hline Characteristics & $\begin{array}{l}\text { All patients } \\
(n=102)\end{array}$ & $\begin{array}{l}\text { Patients with } \\
\text { mild symptoms } \\
\quad(n=43)\end{array}$ & $\begin{array}{c}\text { Survivors with } \\
\text { severe symptoms } \\
(n=39)\end{array}$ & $\begin{array}{l}\text { Non-survivors } \\
\quad(n=20)\end{array}$ & $p$ \\
\hline Age (years) & $55.3 \pm 17.5$ & $45.2 \pm 16.0$ & $57.6 \pm 12.8$ & $72.5 \pm 13.6$ & $<0.0001$ \\
\hline Sex & & & & & 0.023 \\
\hline Female & $41(40 \%)$ & $24(56 \%)$ & $15(38 \%)$ & $5(25 \%)$ & .. \\
\hline Male & $61(60 \%)$ & $19(44 \%)$ & $27(62 \%)$ & $15(75 \%)$ & .. \\
\hline \multicolumn{6}{|l|}{ Chronic diseases } \\
\hline Hypertension & $26(25.5 \%)$ & $3(7 \%)$ & $12(30.8 \%)$ & $11(55 \%)$ & $<0.0001$ \\
\hline Diabetes & $10(9.8 \%)$ & $1(2.3 \%)$ & $7(17.9 \%)$ & $2(10 \%)$ & 0.047 \\
\hline Cardiovascular diseases & $13(12.7 \%)$ & $1(2.3 \%)$ & $6(15.4 \%)$ & $6(30 \%)$ & 0.005 \\
\hline Chronic obstructive pulmonary disease (COPD) & $4(3.9 \%)$ & $2(4.7 \%)$ & $5(7.8 \%)$ & $1(2.6 \%)$ & 1.000 \\
\hline Malignancy & $7(6.9 \%)$ & $0(0 \%)$ & $3(7.7 \%)$ & $4(20 \%)$ & 0.008 \\
\hline \multicolumn{6}{|l|}{ Main signs and symptoms } \\
\hline Fever & $87(85.3 \%)$ & $41(95.3 \%)$ & $37(94.9 \%)$ & $19(95 \%)$ & 0.016 \\
\hline \multicolumn{6}{|l|}{ Highest temperature $\left({ }^{\circ} \mathrm{C}\right)$} \\
\hline$<37.3$ & $15(14.7 \%)$ & $12(27.9 \%)$ & $2(5.1 \%)$ & $1(5 \%)$ & .. \\
\hline 37.3 to 38.0 & $14(13.7 \%)$ & $7(16.3 \%)$ & $6(15.4 \%)$ & $1(5 \%)$ & .. \\
\hline 38.0 to 39.0 & $45(44.1 \%)$ & $20(46.5 \%)$ & $19(48.7 \%)$ & $6(30 \%)$ & .. \\
\hline$\geqslant 39.0$ & $28(27.5 \%)$ & $4(9.3 \%)$ & $12(30.8 \%)$ & $12(60 \%)$ & .. \\
\hline Cough & $55(53.9 \%)$ & $22(51.2 \%)$ & $21(53.8 \%)$ & $12(60 \%)$ & 0.812 \\
\hline Myalgia or fatigue & $60(58.8 \%)$ & $22(51.2 \%)$ & $25(64.1 \%)$ & $13(65 \%)$ & 0.425 \\
\hline Headache & $10(9.8 \%)$ & $5(11.6 \%)$ & $3(7.7 \%)$ & $2(10 \%)$ & 0.909 \\
\hline Diarrhea & $14(13.7 \%)$ & $4(9.3 \%)$ & $4(10.3 \%)$ & $6(30 \%)$ & 0.093 \\
\hline Dyspnea & $19(18.6 \%)$ & $3(7 \%)$ & $8(20.5 \%)$ & $8(40 \%)$ & 0.006 \\
\hline Respiratory percentage (bpm) & $20.5 \pm 4.2$ & $19.6 \pm 1.4$ & $20.3 \pm 4.6$ & $23.1 \pm 6.2$ & 0.023 \\
\hline \multicolumn{6}{|l|}{ Laboratory findings } \\
\hline Leukopenia $(<3.5$ × 109/L) & $29(28.4 \%)$ & $17(39.5 \%)$ & $12(30.8 \%)$ & $0(0 \%)$ & 0.001 \\
\hline Lymphocyte count $(<1.1 \times 109 / \mathrm{L})$ & $65(63.7 \%)$ & $23(53.5 \%)$ & $28(71.8 \%)$ & $14(70 \%)$ & 0.192 \\
\hline
\end{tabular}

Data are mean $\pm \mathrm{SD}$ or $n(\%)$. COPD $=$ Chronic obstructive pulmonary disease, $\mathrm{bpm}=$ breaths per min; respiratory percentage represented the initial respiratory percentage on admission or on the day when visiting doctor.

The typical pattern of CT values in the range of HU $(-,-750)$ was intralesional cavitation, CT values in the range of $\mathrm{HU}[-750,-300)$ was ground glass opacity (GGO) mainly, CT values in the range of $\mathrm{HU}[-300,50)$ was consolidation mainly, and CT values in the range of $\mathrm{HU}[50,+)$ was microvascular enlargement [14] or fibrosis. Reticular pattern, crazy paving pattern, air bronchogram and pleural thickening might contribute to more than one range [15].

\section{Statistical analysis}

Statistical analyses were performed using Statistical Package for Social Science (SPSS version 19; IBM, New York, USA). Distribution normality was assessed using the Shapiro-Walk test. Continuous variables and categorical variables were presented as mean (SD) and frequency (\%), respectively. We used the Mann-Whitney $U$ test, the Kruskal-Wallis test, Fisher's exact test (for categorical data) or one-way ANOVA (for continuous data) to compare differences between patients with mild symptoms, survivors with severe symptoms and non-survivors where appropriate. We used the non-parametric test to compare differences between Stage-1, Stage-2, Stage-3 and Stage-4. A $p$-value of $<0.05$ was defined as *, a $p$-value of $<0.01$ was defined as ${ }^{* *}$, a $p$-value of $<0.001$ was defined as ${ }^{* * *}$. A three-order polynomial estimation model was also conducted to investigate the slope and baseline in above parameters across different stages in the same group. 

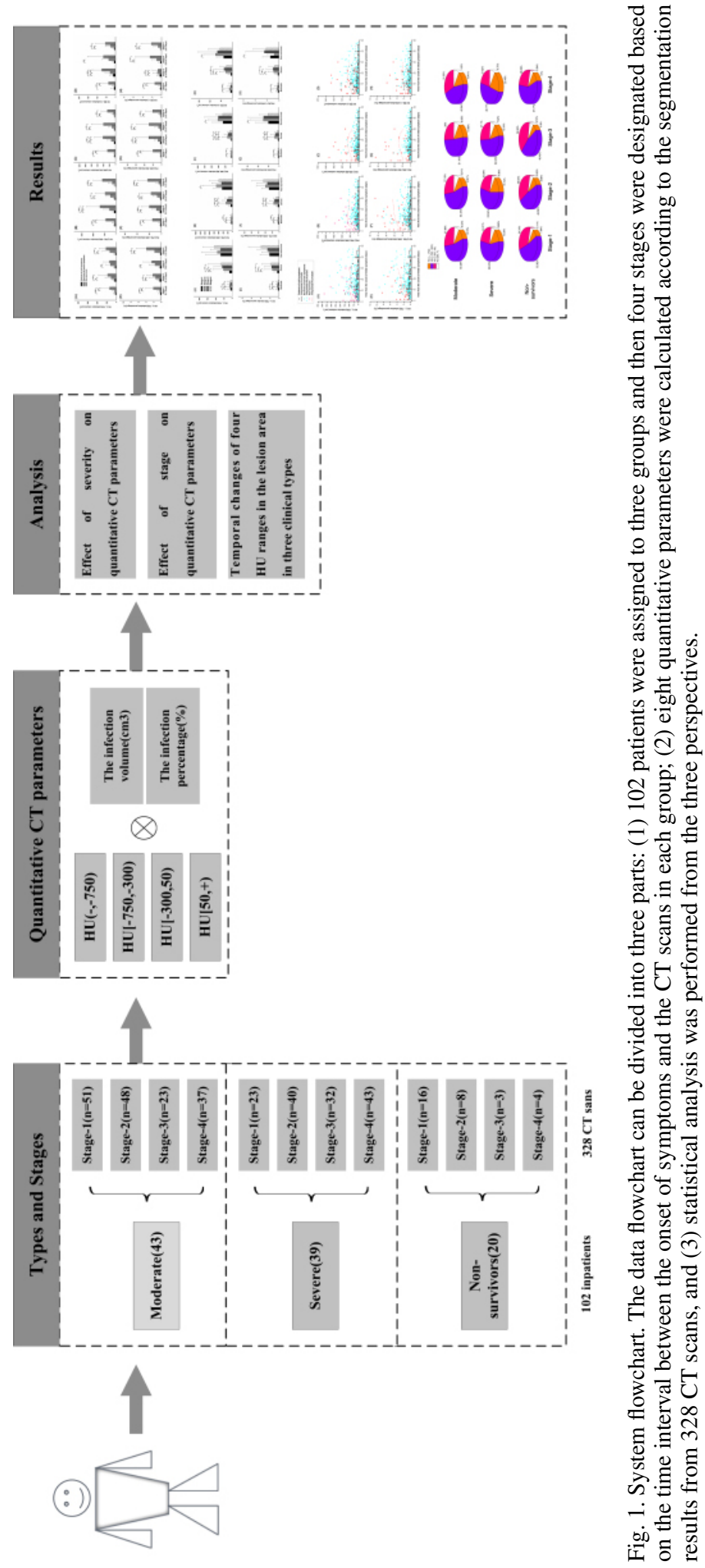


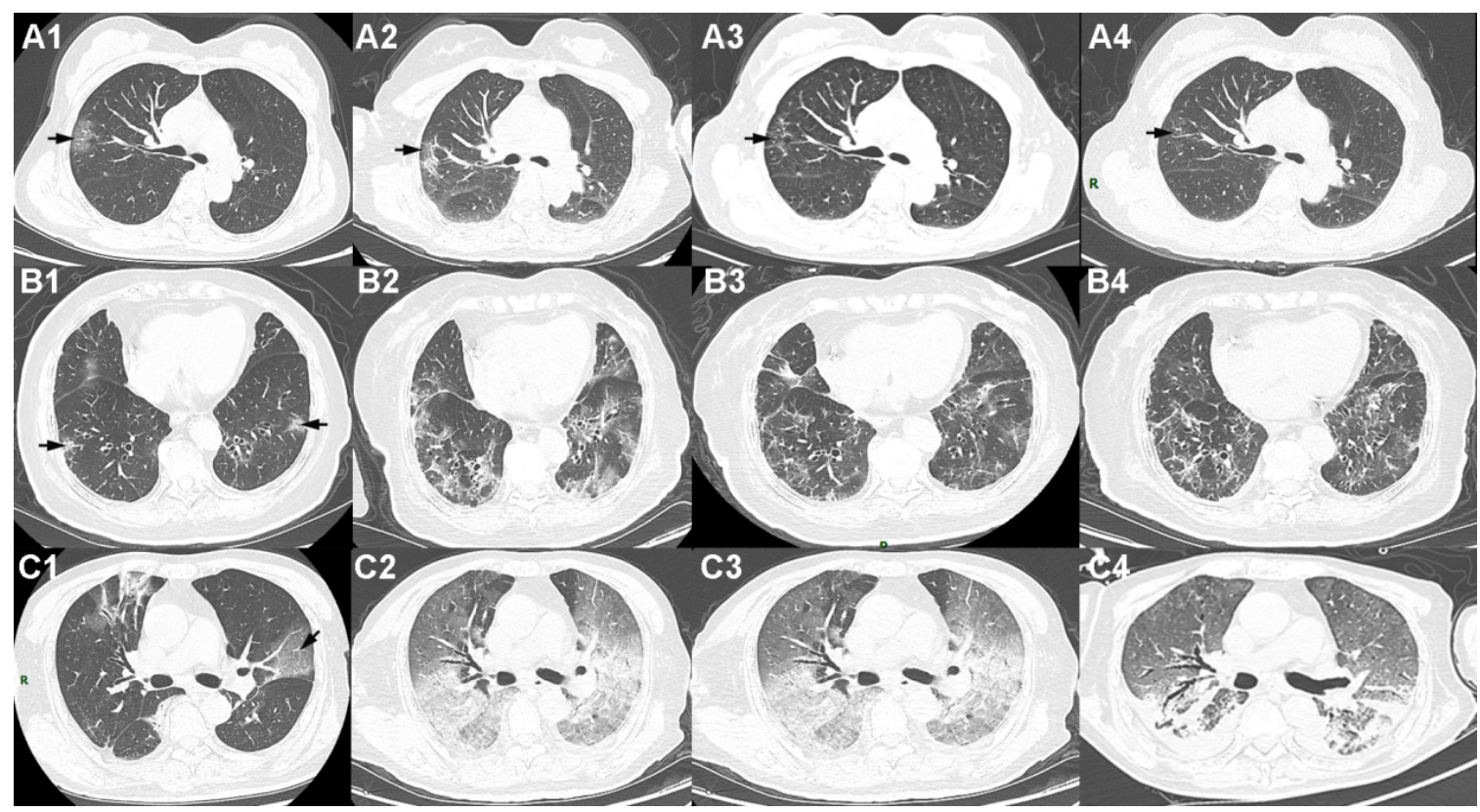

Fig. 2. The CT images of three clinic types of patients at four stages. Shown in A1-A4 (Stage 1-Stage 4) are corresponding slices of a patient with mild symptoms (58 years, male). Shown in B1-B4 (Stage 1-Stage 4) are corresponding slices of a survivor with severe symptoms ( 82 years, female). Shown in C1-C4 (Stage 1-Stage 4) are corresponding slices of a non-survivor (70 years, male). The infection volume was obviously increasing in Stage-2 (8-15 days since symptom onset) in the three patients.

\section{Results}

As shown in Table 1, patients were divided into three clinic types based on severity: 43 (42\%) patients with mild symptoms, $39(38 \%)$ patients with severe symptoms and the remaining $20(20 \%)$ were non-survivors. There were significant differences in age $(p<0.0001)$, sex distribution $(p=0.023)$, hypertension $(p<0.0001)$, cardiovascular disease $(p=0.005)$, diabetes $(p=0.047)$, malignancy $(p=$ $0.008)$, dyspnea $(p=0.006)$ and Leukopenia $(<3.5 \times 109 / \mathrm{L}, p=0.001)$ in three clinic types patients (Table 1).

In Stage-1, there were no differences between patients with mild symptoms and survivors with severe symptoms in the infection volume and percentage in four HU ranges. In Stage-2, there were no differences between survivors with severe symptoms and non-survivors in the infection volume and percentage of $\mathrm{HU}(-,-750)$ and $\mathrm{HU}[-750,-300)$ infection percentage. In Stage-3, there were significant differences between patients with mild symptoms and survivors with severe symptoms in the infection volume and percentage in four HU ranges, meanwhile there were no differences between survivors with severe symptoms and non-survivors in the infection volume and percentage in four HU ranges. In Stage-4, there were no differences between survivors with severe symptoms and non-survivors in the infection volume and percentage in four HU ranges (Fig. 3).

For moderate, there were significant differences in the infection volume and percentage of $\mathrm{HU}(-$, $-750), \mathrm{HU}[-750,-300), \mathrm{HU}[-300,50), \mathrm{HU}[50,+)$ between Stage-2 and Stage-1, Stage-4, and there were no differences in the infection volume and percentage of $\mathrm{HU}(-,-750), \mathrm{HU}[-750,-300), \mathrm{HU}$ $[-300,50), \mathrm{HU}[50,+)$ between Stage-4 and Stage-1, Stage-3, and there were significant differences in the infection volume and percentage of $\mathrm{HU}[-750,-300)$ between Stage- 2 and Stage-3. For severe, there were significant differences in the infection volume and percentage of $\mathrm{HU}(-,-750), \mathrm{HU}[-750$, 


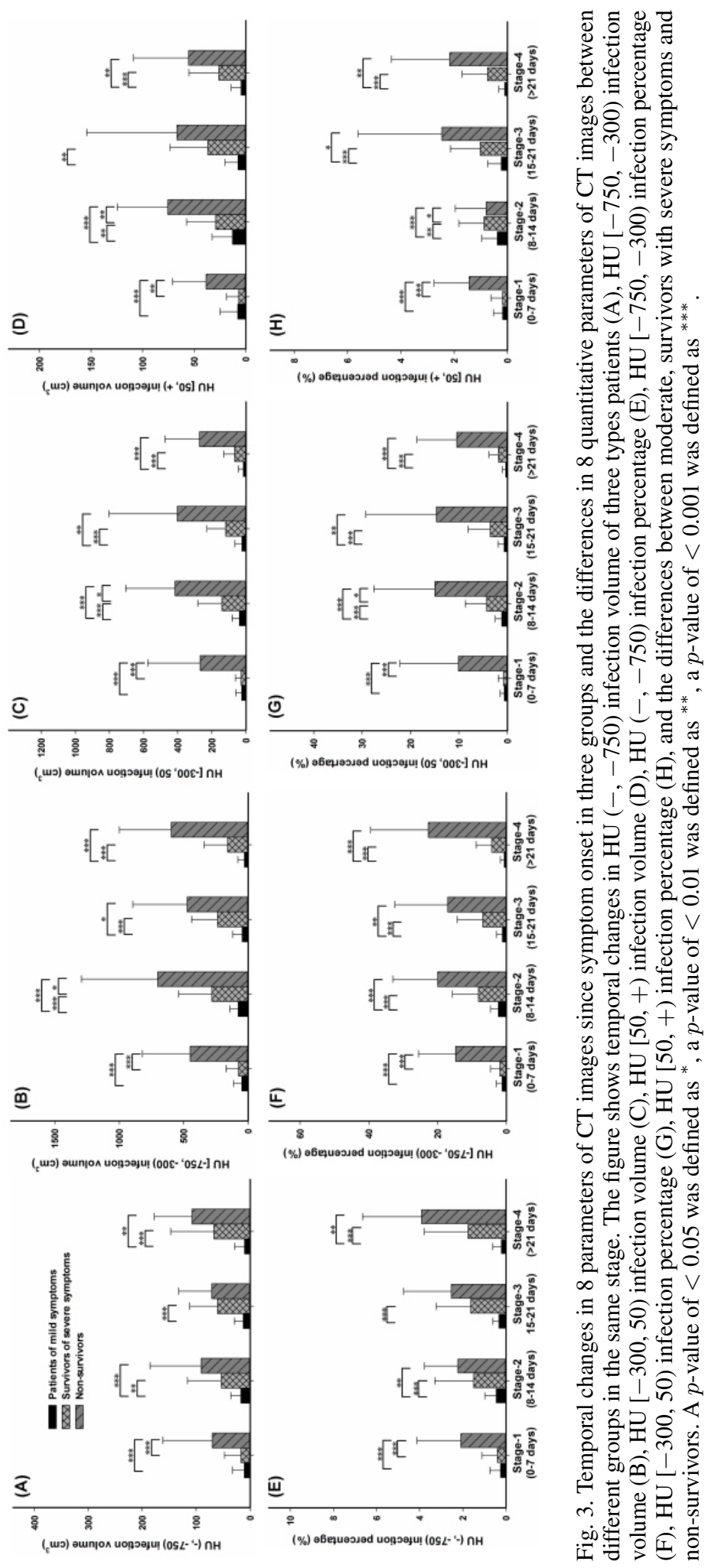




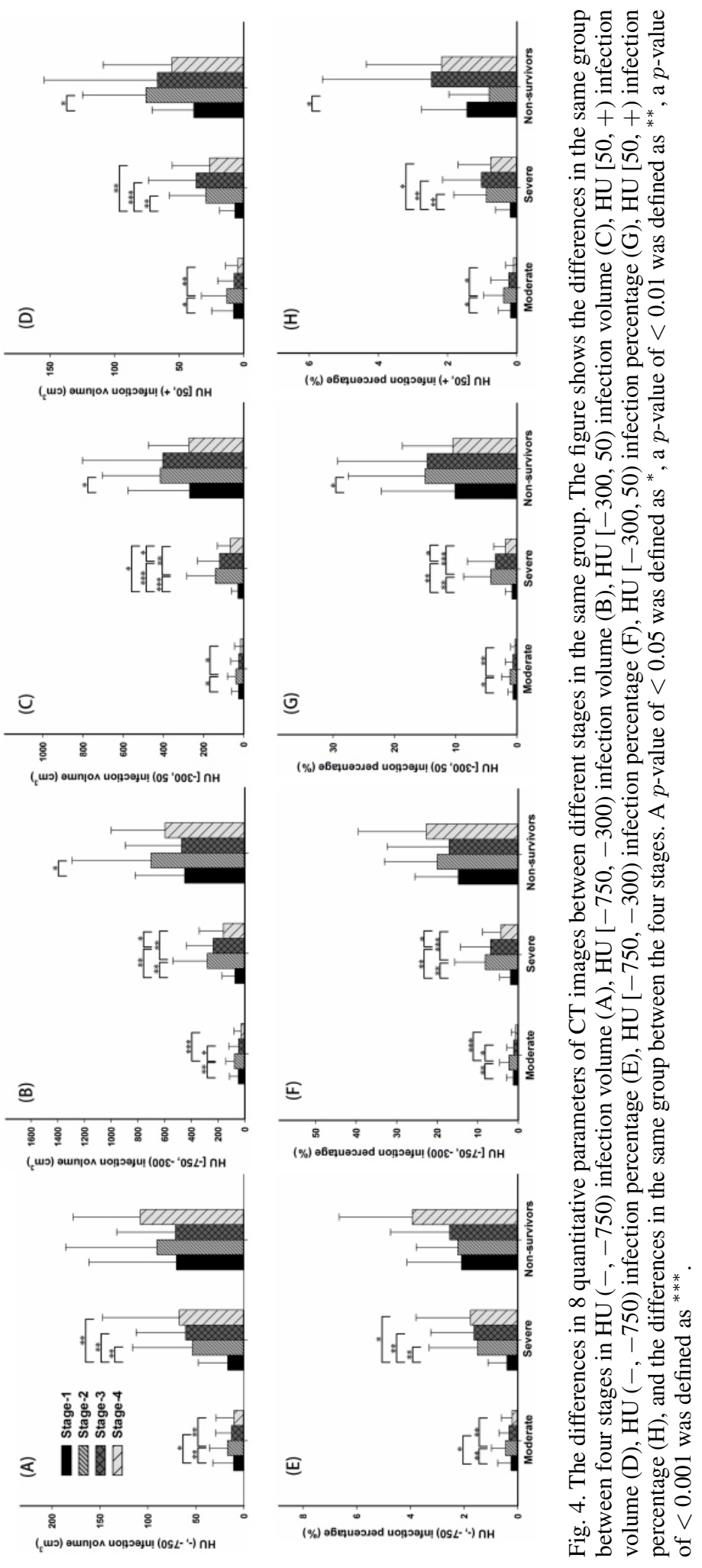




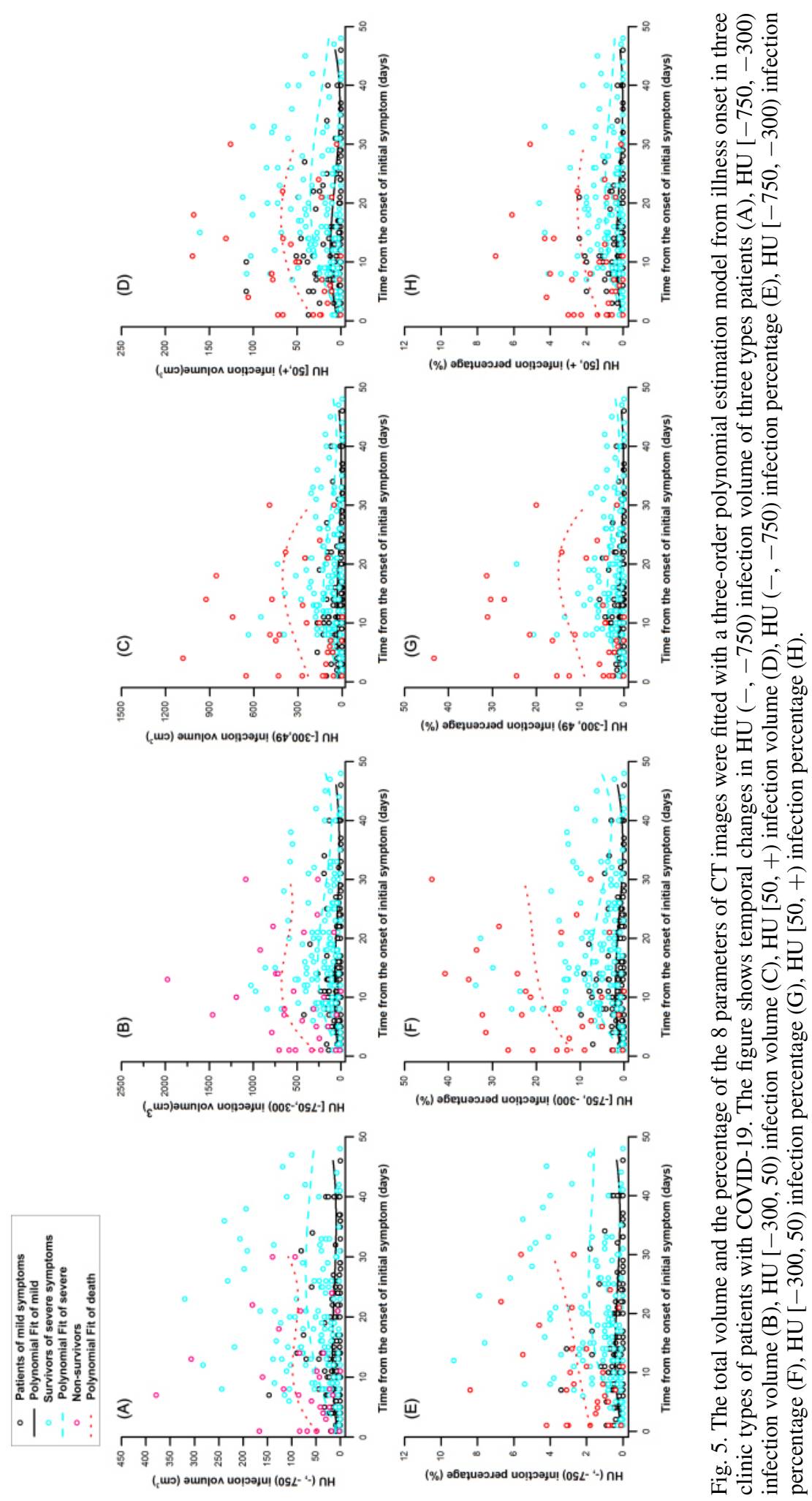




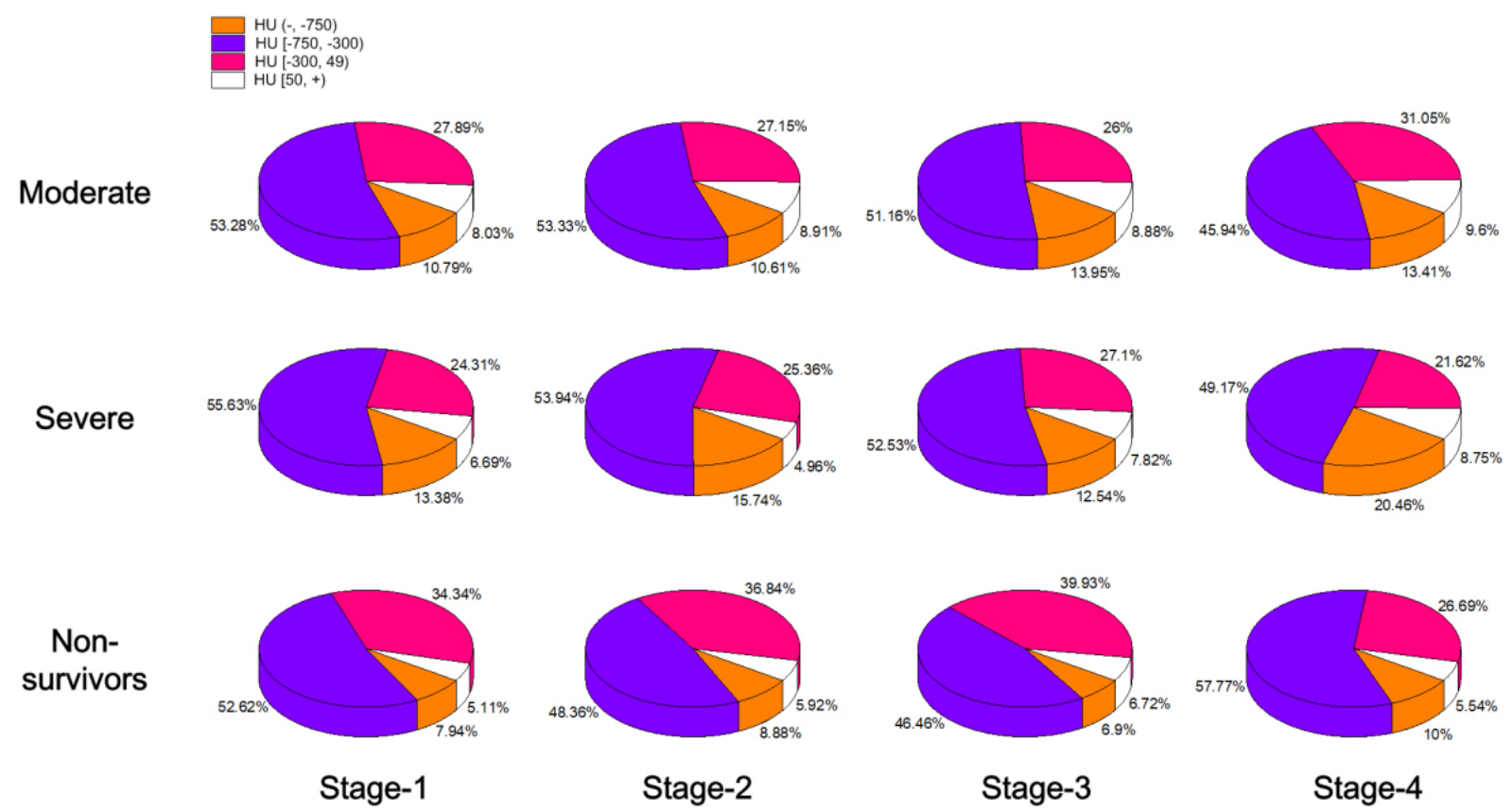

Fig. 6. Temporal changes in the proportion of four HU ranges in the lesion area since symptom onset in three clinic types, moderate (patients with mild symptoms), severe (survivors with severe symptoms) and non-survivors with COVID-19. The 3D color pie chart shows different patterns in proportion in the three groups in four stages.

$-300), \mathrm{HU}[-300,50), \mathrm{HU}[50,+)$ between Stage-1 and Stage-2, Stage-3, and there were significant differences in the infection volume and percentage of $\mathrm{HU}(-,-750)$, HU $[50,+)$, the infection volume of HU $[-750,-300)$ between Stage- 1 and Stage-4, and there were significant differences in the infection volume and percentage of HU $[-750,-300)$, HU $[-300,50)$ between Stage-4 and Stage-2, Stage-3. For non-survivors, there were significant differences in the infection volume and percentage of HU [ -300 , $50), \mathrm{HU}[50,+)$, the infection volume of HU $[-750,-300$ ) between Stage-1 and Stage-2 (Fig. 4).

Figures 3 and 4 show that the infection volume and percentage in four HU ranges were significantly different between the three groups' patients in four stages. The infection volume and percentage of HU $(-,-750), \mathrm{HU}[-750,-300), \mathrm{HU}[-300,50)$ and HU $[50,+)$ were small in Stage-1 (0-7 days from illness onset) and increased significantly in Stage-2 (8-15 days from illness onset) for moderate and severe, and the infection volume and percentage of $\mathrm{HU}(-,-750), \mathrm{HU}[-750,-300), \mathrm{HU}[-300,50)$, HU $[50,+)$ were decreasing in Stage-3 and Stage- 4 for moderate while the 8 parameters had a larger value in four stages for non-survivors. The three-order polynomial estimation model results showed different change trend in three groups (ranked as: moderate $<$ severe $<$ non-survivors) (Fig. 5). The 3D color pie chart shows the highest proportion of $\mathrm{HU}[-300,50)$ in non-survivors among three groups in Stage-1, Stage-2 and Stage-3 (Fig. 6).

\section{Discussion}

This study investigated temporal changes of quantitative CT findings in COVID-19 confirmed patients with different clinic types, including moderate, severe, and non-survivors. The infection volume and percentage of $\mathrm{HU}(-,-750), \mathrm{HU}[-750,-300), \mathrm{HU}[-300,50), \mathrm{HU}[50,+)$ in the total lung were 
extracted from the CT image using artificial intelligence (AI) software. In this study, we investigated the correlation between the 8 quantitative CT parameters in four stages among three clinic patients confirmed with COVID-19 and revealed the cause of deterioration of the COVID-19 patients (Fig. 1).

\subsection{Effect of severity on quantitative CT parameters}

For HU $[-750,-300)$, HU $[-300,50)$ and $\mathrm{HU}[50,+)$, a significant difference was found in infection volume and percentage between non-survivors and patients with mild symptoms in all four stages. Besides, a significant difference was found in the infection volume and percentage of four HU ranges between severe and moderate in Stage-2 and Stage-3. These findings might suggest more involvement in total lung infection in severe than moderate. For HU $(-,-750)$, a significant difference was also found in infection volume and percentage between non-survivors and moderate in Stage-1, Stage-2 and Stage-4. A significant difference was also found in infection volume and percentage between severe and moderate in Stage-2, Stage-3 and Stage-4. These findings indicated more air content in severe group than that in moderate group, which might due to pulmonary edema and hyaline membrane formation [16]. For four HU ranges, a significantly higher infection volume and percentage was found between non-survivors and severe, and between non-survivors and moderate in Stage-1. However, significant differences in these parameters between severe and moderate were only found in Stage-2 and Stage-3. These findings indicate a faster deterioration in non-survivors in Stage-2 and Stage-3 than in the severe and moderate group.

\subsection{Effect of stage on quantitative CT parameters}

For the moderate and severe group, the infection volume and percentage of $\mathrm{HU}(-,-750), \mathrm{HU}[-750$, $-300), \mathrm{HU}[-300,50), \mathrm{HU}[50,+)$ in the total lung reached the maximum value in Stage-2, which indicated that their disease progressed since Stage-2. This finding was consistent with previous studies based on severity scores for each lung or lobes $[12,17]$, which suggested that scores peaked at 6-11 days in CT abnormalities [18] and 10-12 days in chest X-ray abnormalities after symptom onset $[18,19]$. Another study on CT features in 62 patients showed a significantly increased mixed pattern, including GGO, reticular pattern, vacuolar sign, fibrotic streaks, a subpleural line, a subpleural transparent line, air bronchogram, bronchus distortion, and pleural effusion appeared in 8-14 days [19], which is also consistent with our finding (Fig. 2). For severe, the 8 parameters kept at a large valve in Stage-3 and Stage-4, which suggested that a mixed pattern sustained for a period. This might be due to that aggravation and repair might be coexisted at Stage-3, and indicate an irreversible impaired lung function.

\subsection{Temporal changes of four HU ranges in the lesion area in three clinical types}

For moderate, the three-order polynomial estimation model results showed that all 8 parameters had a stable change during course of disease, which was consistent with their good outcome. For severe, the three-order polynomial estimation model results showed that all 8 parameters increased at Stage-1 and Stage-2 and decreased since Stage-3 during course of disease, which implied the rehabilitation. For non-survivors, the three-order polynomial estimation model results that all 8 parameters increased even at Stage-3, which indicated a continuous progression during course of disease. The highest proportion of $\mathrm{HU}[-750,50)(\mathrm{HU}[-750,-300)+\mathrm{HU}[-300,50)>80 \%)$ in the lesion area in non-survivors among three groups indicated that more GGO and consolidation might indicate a poorer prognosis, and quantitative CT parameter of $\mathrm{HU}[-750,50)$ might help us screen severe cases at an early stage. 


\subsection{Limitations}

The sample size of non-survivors is limited and the follow up CT scans of non-survivors were hard to be obtained because of the disease progress, especially in Stage-3 and Stage-4. Besides, without considering the clinic treatment options might lead to a bias in this study and prospective experimental design could eliminate the effect and is a better choice for such research. However, this is unrealistic for the outbreak of COVID-19. In order to make our research conclusion more credible, we will expand the sample size and refine our experimental design according to the preliminary research results in the future.

\subsection{Conclusion}

This study aimed to investigate temporal changes of quantitative CT findings in confirmed COVID19 patients with different clinic types, including moderate, severe, and non-survivors. CT findings of quantitative parameters tend to manifest in different $\mathrm{CT}$ features in different stages from illness onset among different clinic type patients, and the infection volume of four HU ranges were rapidly increased in Stage-1 (the first week) in non-survivors, and peaked in Stage-2 (the second week) in three groups, which indicated that early and timely intervention is very important. Higher proportion of $\mathrm{HU}[-750,50)$ in the lesion area $(\mathrm{HU}[-750,-300)+\mathrm{HU}[-300,50)>80 \%)$ might be a bio-marker for poor prognosis in patients with COVID-19.

\section{Acknowledgments}

The authors thank all their colleagues who helped them with the study. They greatly appreciate the kind assistance of Wang Liang (Radiology, Zhongnan Hospital of Wuhan University, Wuhan, China) in the statistical analysis and the staff Zhang Wei and Shen Hong at Shanghai United Imaging Intelligence Healthcare for technical support. They are also grateful to all medical staff for their great work while risking their lives.

\section{Conflict of interest}

The authors declared that they have no conflicts of interest to this work.

\section{References}

[1] Zhu N, Zhang D, Wang W, Li X, Yang B, Song J, et al. A novel coronavirus from patients with pneumonia in China, 2019. New England Journal of Medicine, 2020.

[2] Cui J, Li F, Shi Z. Origin and evolution of pathogenic coronaviruses. Nature Reviews Microbiology, 2019. 17(3): 181-192.

[3] World Health Organization. 2020. Coronavirus disease (COVID-19) Pandemic. Available from: https://www.who.int/ emergencies/diseases/novel-coronavirus-2019.

[4] Huang C, Wang Y, Li X, Ren L, Zhao J, Hu Y, et al. Clinical features of patients infected with 2019 novel coronavirus in Wuhan, China. The Lancet, 2020. 395(10223): 497-506.

[5] Ai T, Yang Z, Hou H, Zhan C, Chen C, Lv W, et al. Correlation of chest CT and RT-PCR testing in coronavirus disease 2019 (COVID-19) in China: a report of 1014 cases. Radiology, 2020: 200642.

[6] National Health Committee of the People's Republic of China. Notice on Issuing a New Coronavirus Infected Pneumonia Diagnosis and Treatment Plan (Trial Version 5). February 5, 2020. http://www.nhc.gov.cn/yzygj/s7653p/202002/3b09b894 ac9b4204a79db5b8912d4440.shtml. 
[7] McCall B. COVID-19 and artificial intelligence: protecting health-care workers and curbing the spread. The Lancet Digital Health, 2020. 2(4): e166-e167.

[8] Shi H, Han X, Jiang N, Cao Y, Alwalid O, Gu J, et al. Radiological findings from 81 patients with COVID-19 pneumonia in Wuhan, China: a descriptive study. The Lancet Infectious Diseases, 2020.

[9] Lu J, Hu S, Fan R, Liu Z, Yin X, Wang Q, et al. ACP risk grade: a simple mortality index for patients with confirmed or suspected severe acute respiratory syndrome coronavirus 2 disease (COVID-19) during the early stage of outbreak in Wuhan, China. 2020.

[10] Cheng Z, Lu Y, Cao Q, Qin L, Pan Z, Yan F, et al. Clinical features and chest CT manifestations of coronavirus disease 2019 (COVID-19) in a single-center study in Shanghai, China. American Journal of Roentgenology, 2020: 1-6.

[11] China National Health Commission. 2020. Diagnostic and treatment protocol for Novel Coronavirus Pneumonia: Trial version 7. Available from: http://www.nhc.gov.cn/yzygj/s7653p/202003/46c9294a7dfe4cef80dc7f5912eb1989.shtml, (in Chinese).

[12] Shan F, Gao Y, Wang J, Sh W, Shi N, Han M, et al. Lung infection quantification of covid-19 in ct images with deep learning. arXiv preprint arXiv:2003.04655, 2020.

[13] Tang Z, Zhao W, Xie X, Zhong Z, Shi F, Liu J, et al. Severity assessment of COVID-19 using CT image features and laboratory indices. Physics in Medicine \& Biology, 2020.

[14] Caruso D, Zerunian M, Polici M, Pucciarelli F, Polidori T, Rucci C, et al. Chest CT features of COVID-19 in Rome, Italy. Radiology, 2020: 201237.

[15] Ye Z, Zhang Y, Wang Y, Huang Z, Song B. Chest CT manifestations of new coronavirus disease 2019 (COVID-19): a pictorial review. European radiology, 2020: 1-9.

[16] Wang Y, Dong C, Hu Y, Li C, Ren Q, Zhang X, et al. Temporal changes of CT findings in 90 patients with COVID-19 pneumonia: a longitudinal study. Radiology, 2020: 200843.

[17] Wong HYF, Lam HYS, Fong AH, Leung ST, Chin TW, Lo CSY, et al. Frequency and distribution of chest radiographic findings in COVID-19 positive patients. Radiology, 2020: 201160.

[18] Xu Z, Shi L, Wang Y, Zhang J, Huang L, Zhang C, et al. Pathological findings of COVID-19 associated with acute respiratory distress syndrome. The Lancet Respiratory Medicine, 2020. 8(4): 420-422.

[19] Zhou S, Wang Y, Zhu T, Xia L. CT features of coronavirus disease 2019 (COVID-19) pneumonia in 62 patients in Wuhan, China. American Journal of Roentgenology, 2020. 214(6): 1287-1294. 